\title{
ADVANCED MEMS SPATIAL LIGHT MODULATOR FOR COMMUNICATIONS, IMAGING, AND TARGETING
}

\author{
Flavio Pardo, M.E. Simon, V.A. Aksyuk, W.Y-C. Lai, C.S. Pai, F.P. Klemens, J.F. Miner, R.A. Cirelli, \\ E.J. Ferry, J.E. Bower, W.M. Mansfield, A. Kornblit, T.W. Sorsch, J.A. Taylor, M.R. Baker, R. \\ Fullowan, H. Dyson, A. Gasparyan, and S. Arney
}

Bell Labs Lucent Technologies, Murray Hill, NJ USA

\begin{abstract}
$64 \times 64$ arrays of electrostatically actuated piston-tip-tilt micromirrors are realized using a surface micromachining, 3structural-layer polysilicon process. Flat mirrors $(\lambda / 30 @ 1550 \mathrm{~nm}$ wavelength) $120 \mu \mathrm{m}$ in size and fill factor exceeding $98 \%$ have mechanical resonance frequencies of $30 \mathrm{KHz}$ for both tip-tilt and piston motion, with $+/-4$ deg. mechanical tip and tilt and $5 \mu \mathrm{m}$ piston continuous, controllable range under $110 \mathrm{~V}$. The design implements four dual, rotational, in-plane, deep comb drives under each mirror, with $0.5 \mu \mathrm{m}$ critical features.
\end{abstract}

\section{INTRODUCTION}

High speed and high-resolution, ASIC-integrated spatial light modulators (SLM) based on optical MEMS are enabling components for communications, imaging and targeting applications which require precise real-time control of the optical beam's wavefront to achieve maximal, aberration-free performance. Stateof-the-art segmented SLMs are currently limited to about $32 \times 32-$ mirror fast piston-only arrays [1-2], lower pixel count piston-tip-tilt [3], or lower range piston or tilt, 200x240 metal mirror arrays [4]. The combination of piston-tip-tilt in each mirror would improve considerably the phase-reconstruction fidelity [5]. The ultimate specs for such SLMs include high-pixel-count arrays of flat mirrors with 3 degrees of freedom -piston, tip and tilt- with continuous, large displacements and fast response time. The combination of all these characteristics makes the MEMS design and process implementation a formidable challenge. Furthermore, the scalable integration $(64 \times 64$ and larger arrays) with driving electronics puts a severe limit to the

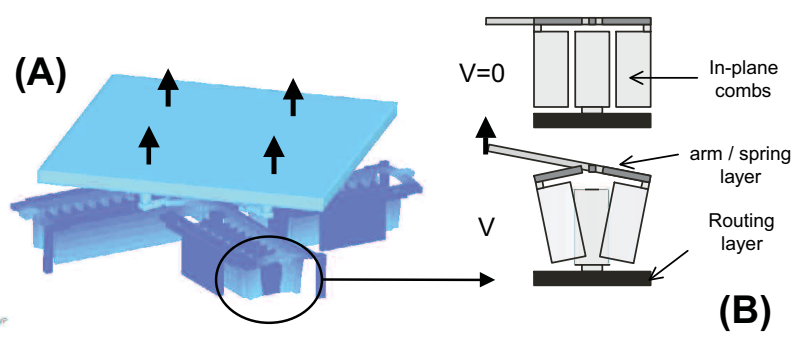

FIGURE 1. Simulation image showing all four dual rotational in plane comb-drives actuated to produce upward piston motion (A) Schematic of the actuation principle (B).

maximum available driving voltage and hence, to the maximum mechanical power that could be achieved in simple, parallel-plate actuators, thus limiting the speed and the range of motion.

\section{MICROMIRROR DESIGN AND PERFORMANCE}

The design solution to the parallel-plate actuator limitations is to exploit the third dimension: deep, dense, in-plane comb-drive actuators. Our basic design is presented in Figure 1. It consists of four dual, rotational, in-plane comb drives, each attached to an arm that rotates out of plane. A flexible joint at the end of each arm attaches to the mirror, allowing 3 independent degrees of freedom of motion: bi-directional tip-tilt, and upward piston. The actuators are densely packed under a high fill factor mirror array.

Figure 2 shows a SEM picture of the finished device characteristics, e.g., the actuators under the mirrosr, back of the
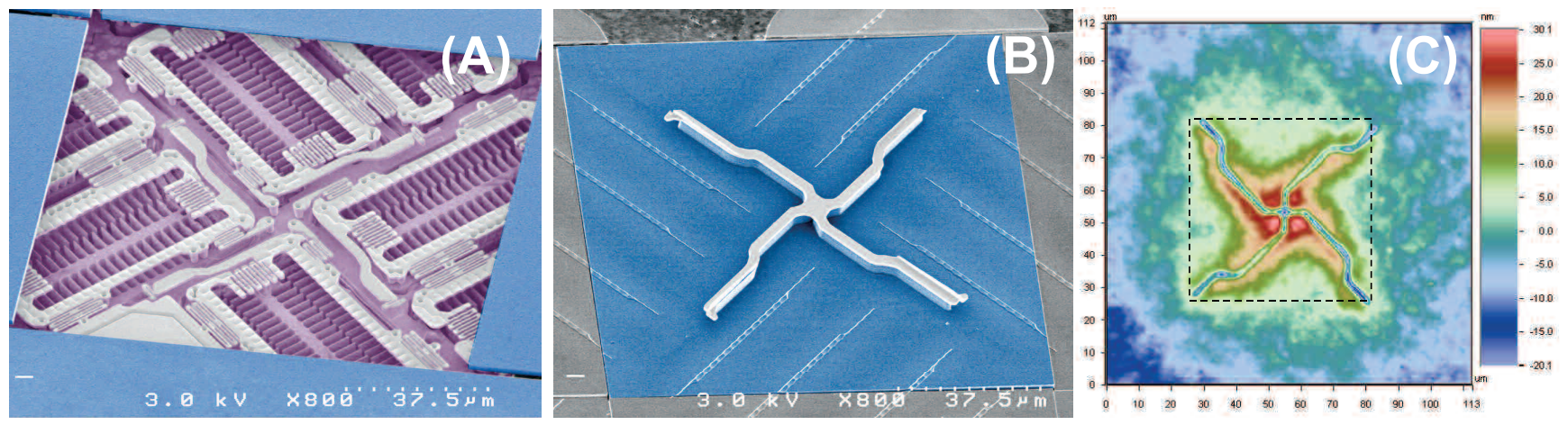

FIGURE 2. SEM image of a finished device (A) showing the four actuators under the mirror and (B) back of the monolithic mirror showing the $4 \mu \mathrm{m}$ thick $X$-shaped strengthening member created by a trench in the sacrificial silicon oxide under it. (C) Interferometer data showing mirror planarity and roughness. Most of the 50nm peak-to-peak topography comes from the non-optimized trench filling process within the central region. The RMS roughness is $8 \mathrm{~nm}$ including the strut print-through and less than $2 \mathrm{~nm}$ without it. The $1.5 \mu \mathrm{m}$ thick mirror has been metalized with $40 \AA \mathrm{Ti} / 400 \AA \mathrm{Au}$ 

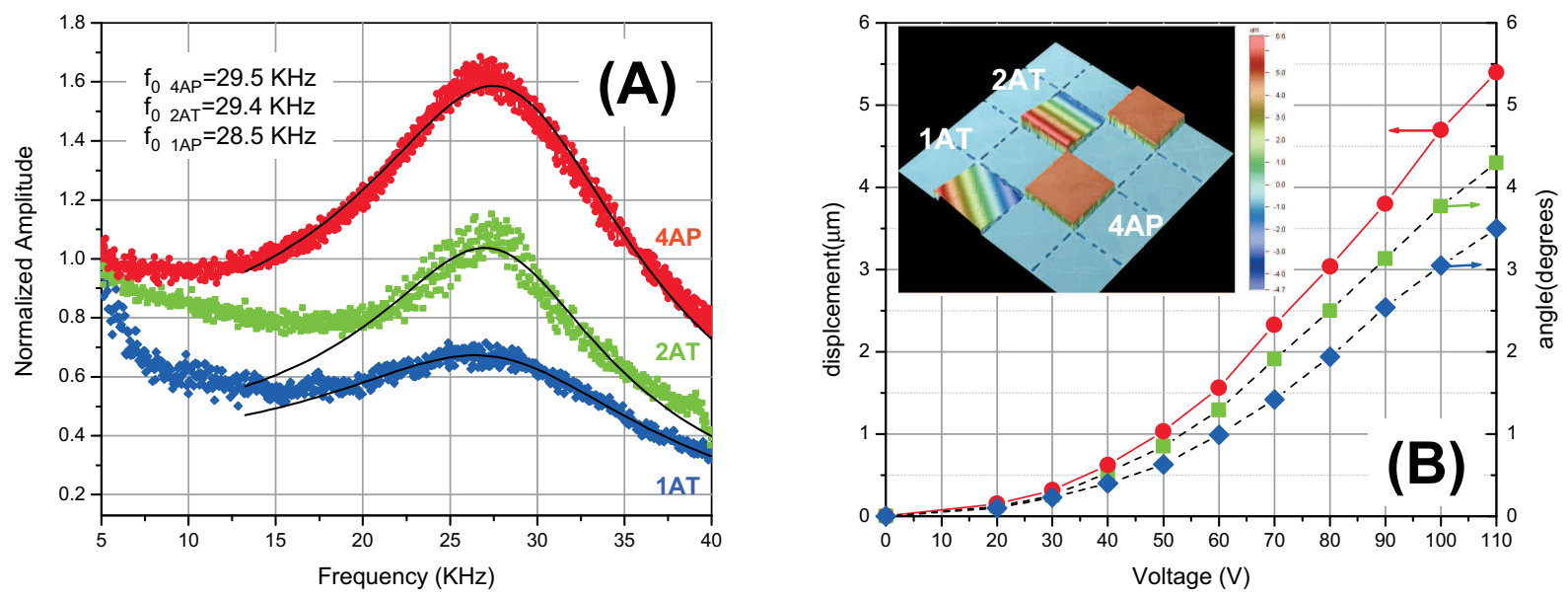

FIGURE 3. (A) Mechanical response of individual mirrors for 60-90V-amplitude tip and piston motion. Experimental data and fitting to driven harmonic system behavior. From the fitting natural frequencies at about $30 \mathrm{kHz}$ are extracted. The responses have a quality factor $Q \sim 2$ at atmospheric pressure, with roll-offs at around $27 \mathrm{KHz}$. (B) Piston and angular displacement versus voltage. Piston is achieved by actuation of all 4 actuators (4AP ). Diagonal tilt $(1 A T \bullet)$ is a one-actuator motion, horizontal or vertical tilt is a two-actuator motion (2AT $\square)$.The inset shows interferometer data for all three actuation modes.

mirror, and front-of-mirror planarity.

Typical dimensions for the most relevant surfacemicromachined polysilicon structural layers are: combs $6 \mu \mathrm{m}$ tall with $0.5 \mu \mathrm{m}$ wide fingers, spring-arm layer thickness of $1-1.5 \mu \mathrm{m}$ with $0.5 \mu \mathrm{m}$ features, and a $1-2 \mu \mathrm{m}$ thick mirror with $0.5-2 \mu \mathrm{m}$ lateral gaps. Low stress silicon dioxide with thickness ranging from 2 to $4 \mu \mathrm{m}$ forms the sacrificial layers between structural layers, for a combined film stack of up to $17 \mu \mathrm{m}$. A critical step in the fabrication is the silicon dioxide filling and planarization of the high-aspectratio combs, achieved by a controlled etch-back, re-deposition step and chemical-mechanical polishing (CMP). The final polysilicon layer forming the mirror is also partially planarized by a CMP touchup to achieve a flat, smooth surface. The structures are released in vapor-phase-HF or in liquid $\mathrm{HF}(49 \%)$ and subsequently dried in a supercritical point $\mathrm{CO}_{2}$ system. A blanket $40 \AA \mathrm{Ti} / 400 \AA \mathrm{Au}$ reflective layer evaporation completes the device. After these steps typically only 1 or 2 mirrors have been observed optically to be stuck or damaged, out of the 4096 in early implementation of the process.

This novel design has been implemented for both $64 \times 64$ and $256 \times 256$ array sizes in a 10 -mask process using $248 \mathrm{~nm}$ stepper optical lithography, which provides uniform exposure of sub-micron features across the array. Special care has been taken in the design of alignment features throughout the process flow to guarantee alignment accuracy better than $100 \mathrm{~nm}$ between all layers.

This structure is currently being monolithically fabricated on a wafer with Through-Wafer-Vias (TWV) to provide electrical connection to the driving electronics chip (to be presented elsewhere). Results presented here were obtained in a hard-wired system, with an extra wiring layer replacing the TWVs and grouping the mirrors in clusters of up to 64 elements.

Figure 3 shows the mechanical response of fabricated devices. The resonance curves were fitted to a driven oscillator model in the peak region to extract resonance frequencies of about $30 \mathrm{kHz}$ and Q 2 for all three degrees of freedom, very close to the FEA simulated values of $29.6 \mathrm{KHz}$ for tilt and $30.9 \mathrm{KHz}$ for piston. The lower frequency roll-offs are due to frequency-dependent damping processes and will require further analysis. Note also the slight inflection point in the angle/displacement versus voltage curves showing a sub-quadratic behavior, a result of the onset of partial disengagement in the rotational comb-drives.

\section{CONCLUSIONS}

We have presented an integration-ready, scalable MEMS SLM mirror array. This work demonstrates a level of complexity never achieved before in terms of the combination of the number of degrees of freedom per chip, pixel speed, controlled motion range and optical quality. Within this MEMS process platform, simulations show that we can achieve $256 \times 256$ arrays with a spec of $10 \mu$ s response time by increasing the spring stiffness (spring layer $\sim 2 \mu \mathrm{m}$ ) and increasing the actuator comb force by fabricating deeper combs of $12 \mu \mathrm{m}$, both well within our fabrication capabilities.

\section{AKNOWLEDGMENTS}

This material is based on work supported by DARPA's CCIT program under contract Number HR0011-04-C-0048 and approved for Public Release, Distribution Unlimited.

\section{REFERENCES}

[1] "High Resolution Wavefront Control Using Micromirror Arrays", Technical Digest of the 2004 Solid-State Sensor, Actuator and Microsystems Workshop, Hilton Head Island, SC, June 6-10, 2004

[2] T. G. Bifano and J. B. Stewart, "High-speed wavefront control using MEMS micromirrors" Proceedings SPIE, 5895 (2005)

[3] J. B. Stewart, T. G. Bifano, P. Bierden, S. Corneliss, T. Cook, B. M. Levine, "Design and development of a 329-segment tip-tilt piston mirror array for space-based adaptive optics" presented at Photonics West, San Jose, CA, January, 2006

[4] A. Gehner, M. Wildenhain, H. Neumann, A. Elgner and H. Schenk. "MEMS Phase Former Kit for High-Resolution Wavefront Control", Proceedings SPIE 5894 (2005).

[5] F. Roddier, "Adaptive Optics in Astronomy" Cambridge University Press, 1999. 